\title{
Paroxysmal Bilateral Dysosmia Treated by Resection of the Olfactory Bulbs
}

\author{
J.M. Markert, M.D., D.O. Hartshorn, M.D., and S.M. Farhat, M.D. \\ Department of Surgery, Section of Neurosurgery and Department of Otolaryngology, Head and Neck Surgery, \\ University of Michigan Medical Center, and Catherine McAuley Health Center, Ann Arbor, Michigan
}

Markert JM, Hartshorn DO, Farhat SM. Paroxysmal bilateral dysosmia treated by resection of the olfactory bulbs. Surg Neurol $1993 ; 40: 160-3$.

Dysosmia, or the distortion of olfaction, is most commonly preceded by viral illness or head trauma, but has a variety of etiologies. The precise nature of the disease process remains obscure. Medical management is largely empiric, and has been aimed at treating underlying illnesses, restricting triggering medications, as well as various pharmacologic interventions. Successful eradication of a severe case of persistent unilateral paroxysmal dysosmia with resection of the ipsilateral olfactory bulb has been reported. We report here a case of bilateral paroxysmal dysosmia, refractory to medical therapy, successfully treated with bilateral olfactory bulb resection.

KEY WORDS: Dysosmia; Parosmia; Olfactory bulb; Cranial nerve syndrome; Olfactory hallucinations

Dysosmia, or parosmia as it is also known, is the distortion or perversion of the sense of smell most commonly seen after head trauma or viral illness $[1,2]$. Olfactory hallucinations are sensations of odors, usually foul, that are frequently associated with temporal or uncinate lobe seizures or psychiatric illness. Frequent overlap occurs between the two disorders. Paroxysmal dysosmia has been described elsewhere, and paroxysmal unilateral dysosmia successfully treated with ipsilateral resection of the olfactory bulk has also been reported [6]. Here we add to the limited literature currently available on this topic with a case report of a patient who, after suffering many years from bilateral paroxysmal dysosmia, was successfully treated with bilateral resection of the olfactory bulbs.

Address reprint requests to: J.M. Markert, M.D., Section of Neurosurgery, University of Michigan Medical Center, Taubman 2128, Ann Arbor, Michigan 48109-0338.

Received July 24, 1992; accepted August 13, 1992.

\section{Case Report}

P.G., a 37-year-old woman, was seen in November 1988. She reported a 17 year history of dysosmia with paroxysms of peculiar sensations of smell. These sensations were usually brought on by inhalation of other strong odors, such as cigars, perfume, or onions; however, they also could be triggered by sudden turning of the head, a big sniff, or emotional and physical stress. The common cold or flu would also exacerbate the sensation. Dysosmia paroxysms were described as unlike any other odor, and always the same: of a hot, foul and smoky nature on the right, and a sickeningly sweet, skunk-like odor on the left. Occasionally the episodes would lateralize to a single side, but in general she experienced both sensations, on their respective sides, with each attack. Dysgeusia, or distortion of taste, did not seem to play a major role in the paroxysms, although she noted tastes compatible with the sensations of smell. The sensations were so powerful that she could not conduct any other activity during an attack, and had to simply rest until they subsided. Resting with her head in a dependent position, with resultant lacrimation, would occasionally relieve her symptoms. These episodes have increased in severity and frequency progressively over the years, and her ability to cope with them has declined.

The patient's past medical history is significant for an episode of influenza during the Asian pandemic of 1968-69 which preceded the onset of her dysosmia paroxysms by approximately three months. She additionally reports being struck in the head by two falling bricks at age seven or eight, apparently sustaining a brief loss of consciousness but no other sequelae. She suffered frequent episodes of otitis media as a child, with placement of bilateral myringotomy tubes. She smoked one pack of cigarettes per day for approximately three years, but stopped smoking in 1973. She has a history of hypothyroidism for which she is receiving replacement therapy.

The patient sought help for her condition for many years. She was seen at the Taste and Smell Clinic of Georgetown University Medical Center where work-up 
was done, including sinus and skull radiographs, cranial computerized tomography as well as magnetic resonance imaging; all were negative. She also had several electroencephalograms during dysosmic paroxysms, all of which were negative. A repeat EEG showed minimal intermittent electrical disturbance in cerebral activity, which was not felt to be significant, and no evidence of epileptiform activity. Intranasal electrode EEG was also negative. She was later evaluated at the University of Connecticut Taste and Smell Center and found to have minimal septal deviation, no intranasal disease, and mild unilateral hyposmia on the left side $(70 \%$ of normal function). She was normosmic on the right with $90 \%$ of normal function. Taste was normal. Electrolytes, SGOT, SGPT, IgE, CBC with differential, and urinalysis were all within normal limits. Thyroid function tests demonstrated only a mild elevation in T4. No cause for her disorder was identified. She was treated with various medical regimens, including phenytoin, phenobarbitol, thioridazine, and haloperidol, propranolol, depakene, and papaverin. Mild improvement was noted only with the haloperidol, and only at doses that resulted in excessive somnolence. She was also treated with intranasal corticosteroids and zinc supplementation without significant improvement.

Based on an earlier report of a similar unilateral case [6], we offered the patient the option of a craniotomy with bilateral resection of the olfactory bulbs. In January 1990 , she underwent the proposed operation. Although a pterional approach was initially considered, it was felt that a transfrontal approach would give optimal exposure. A single bone flap was raised in the frontal region across the midline. The dura was opened bilaterally and the superior sagittal sinus left intact. The right frontal lobe was retracted laterally, and its medial aspect followed down to the underlying cribriform plate. The large, pale yellow olfactory bulb, normal in appearance, was immediately noted and resected. The left frontal lobe was then retracted laterally with resection of that olfactory bulb undertaken in an identical fashion with similar findings. Microscopic examination was normal, with no evidence of tumor, inflammation, vasculitis, amyloid, or other conditions.

The patient recovered from her operation without incident. She noted complete anosmia postoperatively, with total relief of her symptoms immediately. Noxious challenges similar to those which would have immediately induced dysosmia preoperatively had no effect. She has been followed for 15 months without recurrence of her symptoms.

\section{Discussion}

Approximately 2 million Americans suffer from disorders of taste and smell, according to a 1979 study re-
Table 1. Disorders of Smell (3)

\begin{tabular}{ll}
\hline $\begin{array}{l}\text { Quantitative } \\
\text { Anosmia: } \\
\text { Hyposmia: }\end{array}$ & $\begin{array}{c}\text { inability to detect odors } \\
\text { decreased ability to detect } \\
\text { odors } \\
\text { decreased threshold for odor } \\
\text { detection }\end{array}$ \\
$\begin{array}{l}\text { Hyperosmia: } \\
\text { Qualitative }\end{array}$ & $\begin{array}{l}\text { distortion of odor perception } \\
\text { Dysosmia or Parosmia: }\end{array}$ \\
$\begin{array}{l}\text { Cacosmia: } \\
\text { Phantosmia: }\end{array}$ & $\begin{array}{l}\text { as malodorous } \\
\text { hallucination of odors } \\
\text { abnormal inability to distinguish } \\
\text { between odors }\end{array}$ \\
\hline
\end{tabular}

ported by the National Advisory of Neurological and Communicative Disorders and Stroke Council [16]. Disorders of smell are classified as "-osmias." The nomenclature in the literature is inconsistent; however, Table 1 gives a general overview. In addition, these disorders are subclassified as general (affecting all odors), partial (affecting some, but not all), or specific (affecting only one or a very limited number). They may be either constant or intermittent in nature $\{2,3,15\}$ (Table 1 ).

The anatomic origin for abnormalities in smell perception may be peripheral, central or combined. Peripheral abnormalities may arise at the level of the nasal mucosa or the olfactory nerves. Central abnormalities commence at the level of the olfactory bulb, which may function as a primitive locus for limited odor discrimination. Continuous background electrical activity in the bulbs is increased by olfactory stimulation. The olfactory tracts become medial and lateral striae which travel to the cerebral hemispheres bilaterally; there is no thalamic relay. The olfactory cortex in man occupies only a small portion of each anteriomedial temporal lobe, the pyriform cortex [1].

Dysosmias have also been classified as central or peripheral in nature [15]. Central dysosmias include: (1) illusions of smell perceived in certain medical conditions including pregnancy; (2) olfactory hallucinations as experienced by psychotic patients; and (3) uncinate fits, or hallucinations of smell associated with temporal lobe seizures. Peripheral dysosmias include cacosmia, constant or periodic episodes of bad smell resulting from abnormalities of the upper airway and nose or from inflammatory conditions such as sinusitis or tonsillitis. Essential parosmia exists when stimulation of the sense of smell results in a perverted sensation, usually of a foul odor. It can result from infections, cranial injury, or exposure to either systemic or local toxins. Most cases of essential parosmia, however, are of unknown etiology. This disorder has been compared to trigeminal neuralgia and other cranial nerve paroxysmal syndromes; as it 
shares many of the common features of these conditions, including precipitating stimulus (usually of a mechanical nature), characteristically brief attacks, and a cranial nerve which may or may not be demonstrably damaged [6].

Dysosmia and anosmia have various etiologies. The single most common cause of disorders of smell is a viral infectious syndrome [2]. Many of these disorders have been traced to specific influenza pandemics, notably to the "Hong-Kong" flu pandemic of 1968-69 [4]. Other infections associated with subsequent dysosmia or hyposmia include meningitis, viral hepatitis, polio, yellow fever, smallpox, typhoid fever, diphtheria, plague, measles, and impetigo. Return of normal function is often noted after several weeks or months.

Blunt head trauma is a frequent cause of these disorders, with loss of smell presenting more frequently than its distortion. The putative mechanism involves shearing of the olfactory nerves at the cribriform plate; subsequent axonal regeneration is usually minimal, and reconnection of receptor cells with the olfactory bulb further hampered by scarring $[1,5]$. Recovery of normal function may occur after weeks or months, but frequently damage is permanent.

Tumors or other irritative lesions can lead to a dysfunction of the sense of smell. Meningiomas are the most commonly involved intracranial tumors, particularly when located along the suprasellar region, sphenoid ridge, frontal parasagittal region, or the olfactory groove [2]. Other intracranial tumors that can affect olfaction include gliomas, craniopharyngiomas, neurofibromas, schwannomas, xanthogranulomas, tuberculomas, dermoids, encephaloceles, and large aneurysms of the internal carotid or anterior communicating arteries. Hydrocephalus forcing the floor of the third ventricle downward can also account for such dysfunction $[2,3,7,12]$. Intracranial tumors within the temporal lobe, or impinging on it, can cause olfactory hallucinations with an incidence approaching $20 \%$. Many of these tumors are associated with temporal lobe epilepsy and uncinate fits. Olfactory hallucinations have also been reported with lesions in other areas of the brain acting through association bundles and thus triggering the temporal lobe syndrome [14].

Psychiatric disorders are associated with olfactory hallucinations, most commonly with major depressive episodes, schizophrenia, confusional states, and acute psychosis. In general, the perceived odor is unpleasant. Recognition of these hallucinations has apparently increased in recent years with a heightened level of awareness amongst mental health professionals, and indeed, dysosmia is now recognized as a potential mask of depressive illness $[2,9]$.
Previous surgery or radiation can result in disorders of the sense of smell, most frequently hyposmia. These are usually associated with therapy directed at the nasal region. Laryngectomy can decrease the acuity of the sense of smell by decreasing the active passage of air through the nose, and preventing adequate stimulation of the olfactory epithelium $[2,11]$. Coronary bypass has also been associated with parosmia [10].

Drugs are another frequent offender; clofibrate, amphotericin B, carbimazole, calcium channel blockers, and many other medications have been associated with dysosmia. Many chemicals and organic solvents have also been linked to cacosmia $[2,5,8,9,13]$.

Systemic illnesses such as diabetes, chronic renal disease, cirrhosis, malnutrition, Addison's disease, congenital adrenal hyperplasia, hypothyroidism, and various other endocrine abnormalities and autoimmune syndromes can also distort the sense of smell $[2,3]$.

A lack of understanding of the precise pathophysiologic processes contributing to dysosmias and the disparity of the underlying etiologies renders treatment of these disorders obscure. Some success has been obtained from treatment of an underlying medical condition or discontinuing contributing medications. Pharmacotherapy has been widely empiric; our patient was treated with a variety of medications including anticonvulsants, antipsychotics, steroids, beta blockers, and corticosteroids, all without success. Until a better understanding of this disease process exists, surgical intervention may be appropriate for treatment of selected severe cases of this condition not responsive to medical and pharmacologic management.

\section{Conclusion}

Disorders of smell, including the syndrome of parosmia, may have many clinical etiologies. These must be carefully evaluated, as they can be clues to other illnesses or lesions. Many cases will remain obscure in origin, but even these usually remit in a matter of months. For the patient with persistent, severe paroxysmal dyosmia of unknown origin, refractory to medical management, surgical sectioning of the olfactory bulk has been shown to relieve the condition in at least one unilateral case and can now be viewed as a potential therapeutic option for the bilateral occurrence as well.

\section{References}

1. Adams RD, Victor M: Disorders of smell and taste. In: Principles of neurology. New York, McGraw Hill, 1989:181-9. 
2. Doty RL: A review of olfactory dysfunction in man. Am J Otolaryngol 1979;1:57-79.

3. Estrem SA, Renner G: Disorders of smell and taste. Oto Clin NA 1987;20:133-47.

4. Henkin RI, Larson AL, Powell RD: Hypogeusia, dysgeusia, hyposmia, and dysosmia following influenza-like infection. Ann Otol Rhinol Laryngol 1975;84:672-82.

5. Jafek BW, Eller PM, Esses BA, Moran DT: Post-traumatic anosmia: ultrastructural correlates, Arch Neurol 1989;46:300-4.

6. Kaufman MD, Lassiter KR, Shenoy BV: Paroxysmal unilateral dysosmia: a cured patient. Ann Neurol 1988;24:450-1.

7. Kinjo T, Mukawa J, Miyaqi K, Takara E, Mekaru S, Ishikawa Y: A case of calcified intracranial tuberculoma present unique MRI findings.' No Shinkei Geka 1988;16:791-5.

8. Levenson JL, Kennedy K: Letters and corrections: dysosmia, dysgeusia, and nifedipine. Ann Int Med 1985;102:135-6.

9. Levenson JL: Dysosmia and dysgeusia presenting as depression. Gen Hosp Psychiatry 1985;7:171-3.
10. Mohr PD: Correspondence: early neurological complications of coronary artery bypass surgery. Br Med J 1986;292:60.

11. Ritter FN: Fate of olfaction after laryngectomy. Arch Otolaryngol 1969;79:169-71

12. Rutherford GS, Mathew B: Xanthogranuloma of the choroid plexus of the lateral ventricle, presenting with parosmia and parageusia. Br J Neurosurg 1987;1:285-8.

13. Ryan CM, Morrow CA, Hodgson M: Brief communication: cacosmia and neurobehavioral dysfunction associated with occupational exposure to mixtures of organic solvents. Am J Psychiatry $1988 ; 145: 1442-5$.

14. Schhneider RC, Crosby EC, Farhat SM: Extratemporal lesions triggering the temporal-lobe syndrome: the role of association bundles. J Neurosurg 1965;22:246-63.

15. Zilstorff D, Herbild O: Parosmia. Acta Otolaryngol Supp 1979; 360:40-1.

16. Ziporyn T: Taste and smell: the neglected sense. JAMA 1982; 247:277-85. 